\title{
On approximate solutions for two higher-order Caputo-Fabrizio fractional integro-differential equations
}

\author{
S Melike Aydogan', Dumitru Baleanu ${ }^{2,3^{*}}$, Asef Mousalou ${ }^{4}$ and Shahram Rezapour ${ }^{4}$
}

\section{"Correspondence:}

dumitru@cankaya.edu.tr

${ }^{2}$ Department of Mathematics,

Cankaya University, Ogretmenler

Cad. 14, Balgat, Ankara, 06530,

Turkey

${ }^{3}$ Institute of Space Sciences, Magurele, Bucharest, Romania Full list of author information is available at the end of the article

\begin{abstract}
We investigate the existence of solutions for two high-order fractional differential equations including the Caputo-Fabrizio derivative. In this way, we introduce some new tools for obtaining solutions for the high-order equations. Also, we discuss two illustrative examples to confirm the reported results. In this way one gets the possibility of utilizing some continuous or discontinuous mappings as coefficients in the fractional differential equations of higher order.
\end{abstract}

MSC: 34A08; 34A99

Keywords: approximate fixed point; higher-order fractional differential equation; non-singular kernel; Caputo-Fabrizio derivation

\section{Introduction}

Up to now, there have been defined some fractional derivations of which most used are the Caputo and Riemann-Liouville operators. The applications of the fractional calculus with these two main derivatives can be observed within a huge range of real world phenomena. In order to increase the power and applicability of the fractional calculus some researchers suggested a new type of fractional derivatives possessing different kernels. Thus, Caputo and Fabrizio defined recently a new fractional derivative possessing a singular kernel [8] and the properties of it were discussed in [11]. Some researchers have used distinct methods for solving some different equations including the Caputo-Fabrizio (CF) fractional derivative (see $[1-7,9,10]$ and the references therein) and multi-singular pointwise defined equations [13-16]. Despite these original results, still several issues regarding this new fractional derivative have to be developed. Below we discuss the existence of approximate solutions analytically corresponding to two CF fractional differential equations (FDE).

The plan of the manuscript can be seen below. In the following section we recall the main results needed in this paper. Section 3 contains the original results and the illustrative examples. Finally, Section 4 summarizes our work.

\section{Basic tools}

Let $c>0, u \in H^{1}(0, c)$ and $\alpha \in(0,1)$. We recall that ${ }^{C F} D^{\alpha} u(t)=\frac{(2-\alpha) M(\alpha)}{2(1-\alpha)} \int_{0}^{t} \exp \left(\frac{-\alpha}{1-\alpha}(t-\right.$ $r)) u^{\prime}(r) d r$ means the CF fractional derivative, such that $t \geq 0$ and $M(\alpha)$ denotes the nor-

(c) The Author(s) 2017. This article is distributed under the terms of the Creative Commons Attribution 4.0 International License (http://creativecommons.org/licenses/by/4.0/), which permits unrestricted use, distribution, and reproduction in any medium, provided you give appropriate credit to the original author(s) and the source, provide a link to the Creative Commons license, and indicate if changes were made. 
malization constant and fulfilling $M(0)=M(1)=1$ [8]. The corresponding fractional integral is written as ${ }^{C F} I^{\alpha} u(t)=\frac{2(1-\alpha)}{(2-\alpha) M(\alpha)} u(t)+\frac{2 \alpha}{(2-\alpha) M(\alpha)} \int_{0}^{t} u(r) d r$ for $0<\alpha<1$ [11]. We recall that $M(\alpha)=\frac{2}{2-\alpha}$ for all $0 \leq \alpha \leq 1$ [11]. As a result, the CF fractional derivative becomes ${ }^{C F} D^{\alpha} u(t)=\frac{1}{1-\alpha} \int_{0}^{t} \exp \left(-\frac{\alpha}{1-\alpha}(t-r)\right) u^{\prime}(r) d r$, where $t \geq 0$ and $0<\alpha<1$ [11]. Note that ${ }^{C F} D^{\alpha} u \in H^{1}$ when $u \in H^{1}$. This hints to a new idea about high-order derivations. If $n \geq 1$ and $\alpha \in[0,1]$, then the CF fractional derivative of order $n+\alpha$ is defined by ${ }^{C F} D^{\alpha+n} u:={ }^{C F} D^{\alpha}\left({ }^{C F} D^{n} u(t)\right)[8]$. We need the following results.

Lemma 2.1 ([11]) The unique solution of ${ }^{C F} D^{\alpha} u(t)=v(t)$ with $u(0)=c$ and $0<\alpha<1$ is given by $u(t)=c+a_{\alpha}(v(t)-v(0))+b_{\alpha} \int_{0}^{t} v(r) d r$, where $a_{\alpha}=\frac{2(1-\alpha)}{(2-\alpha) M(\alpha)}=1-\alpha$ and $b_{\alpha}=$ $\frac{2 \alpha}{(2-\alpha) M(\alpha)}=\alpha$. In addition $v(0)=0$.

For investigating the existence of solutions for most FDE, researchers utilized the welldefined fixed point results, e.g. the Banach contraction principle. We recall that there are many nonlinear FDE admitting no exact solutions [2]. In this case, numerical methods are utilized to get an approximation of exact solutions. In addition, $u$ represents an approximate solution for FDE when we could get a sequence of functions $\left\{v_{n}\right\}_{n \geq 1}$ such that $v_{n} \rightarrow u$.

If an exact solution $u$ is not obtained, then we use this approach. This case arises when we discuss the FDE within a non-complete metric space.

Below we present some basic notions needed in this manuscript. Let $(Y, d)$ denoting a metric space, $F$ a self-map on $Y, \alpha: Y \times Y \rightarrow[0, \infty)$ a mapping and $\varepsilon$ a positive number. We say that $F$ is $\alpha$-admissible when $\alpha(x, y) \geq 1$ implies $\alpha(F x, F y) \geq 1$ [12]. When $d\left(F x_{0}, x_{0}\right) \leq \varepsilon, x_{0} \in Y$ is called an $\varepsilon$-fixed point of $F$.

We say that $F$ possesses the approximate fixed point property when $F$ possesses an $\varepsilon$-fixed point for all $\varepsilon>0$ [12]. We recall that some mappings admit approximate fixed points while possessing no fixed points [12]. Let $\mathcal{R}$ be the set of all continuous mappings $f:[0, \infty)^{5} \rightarrow[0, \infty)$ fulfilling $f(1,1,1,2,0)=f(1,1,1,0,2):=h_{1} \in(0,1)$, $f\left(\mu x_{1}, \mu x_{2}, \mu x_{3}, \mu x_{4}, \mu x_{5}\right) \leq \mu f\left(x_{1}, x_{2}, x_{3}, x_{4}, x_{5}\right)$ for all $\left(x_{1}, x_{2}, x_{3}, x_{4}, x_{5}\right) \in[0, \infty)^{5}$ and $\mu \geq 0$ and also $f\left(x_{1}, x_{2}, x_{3}, 0, x_{4}\right) \leq f\left(y_{1}, y_{2}, y_{3}, 0, y_{4}\right)$ and $f\left(x_{1}, x_{2}, x_{3}, x_{4}, 0\right) \leq f\left(y_{1}, y_{2}, y_{3}, y_{4}, 0\right)$ when $x_{1}, \ldots, x_{4}, y_{1}, \ldots, y_{4} \in[0, \infty)$ with $x_{j}<y_{j}$ for $j=1,2,3,4$ [12]. $F$ denotes a generalized $\alpha$ contractive mapping when there exists $f \in \mathcal{R}$ obeying

$$
\alpha(x, y) d(F x, F y) \leq f(d(x, y), d(x, F x), d(y, F y), d(x, F y), d(y, F x))
$$

for all $x, y \in Y$ [12]. We need the following result.

Theorem 2.2 ([12]) Let $(Y, d)$ denoting a metric space, $\alpha: Y \times Y \rightarrow[0, \infty)$ be a mapping and $F$ denoting a generalized $\alpha$-contractive and $\alpha$-admissible self-map on $Y$. Let us suppose that there exists $x_{0} \in Y$ obeying $\alpha\left(x_{0}, F x_{0}\right) \geq 1$. Then $F$ possesses an approximate fixed point.

\section{The results}

We use the main idea of [6] for obtaining our results in this work. 
As is well known, by using the Cauchy formula for repeated integration, we get

$$
\begin{aligned}
J^{n} u(t) & =\underbrace{\int_{0}^{t=t_{n}} \int_{0}^{s=t_{n-1}} \int_{0}^{t_{n-2}} \cdots \int_{0}^{t_{1}} u\left(t_{0}\right) d t_{0}, d t_{1}, \ldots, d\left(t_{n-2}\right) d s}_{n} \\
& =\frac{1}{(n-1) !} \int_{0}^{t} u(s)(t-s)^{n-1} d s,
\end{aligned}
$$

for all $n \geq 1, a, t \in \mathbb{R}$ and $t>0$. If $n$ is substituted by a positive real number $\alpha$ and $(n-1)$ ! by its generalization $\Gamma(\alpha)$, a formula for fractional integration is obtained for the fractional operator $J^{\alpha} u(t)=\frac{1}{\Gamma(\alpha)} \int_{0}^{t} u(s)(t-s)^{\alpha-1} d s$, which is called the Riemann-Liouville fractional integral of order $\alpha$.

Let us to consider the following symbols:

$$
\begin{gathered}
{ }^{C F} D^{\alpha^{[n]}} u(t):=\underbrace{{ }^{C F} D^{\alpha}\left({ }^{C F} D^{\alpha}\left({ }^{C F} D^{\alpha} \cdots\left({ }^{C F} D^{\alpha} u(t)\right) \cdots\right)\right)}_{n}, \quad{ }^{C F} D^{\alpha}{ }^{[0]} u(t)=u(t), \\
\int_{0}^{t^{[n]}} u(s) d s=\underbrace{\int_{0}^{t=t_{n}} \int_{0}^{s=t_{n-1}} \int_{0}^{t_{n-2}} \cdots \int_{0}^{t_{1}} u\left(t_{0}\right) d t_{0}, d t_{1}, \ldots, d\left(t_{n-2}\right) d s}_{n}=J^{n} u(t),
\end{gathered}
$$

and $J^{0} u(t)=\int_{0}^{t^{[0]}} u(s) d s:=u(t)$. Also, we define

$$
\begin{aligned}
\left(a_{\alpha}+b_{\alpha} J u(t)\right)^{[n]}= & \left(a_{\alpha}+b_{\alpha} \int_{0}^{t} u(s) d s\right)^{[n]} \\
= & \left(\begin{array}{c}
n \\
0
\end{array}\right) a_{\alpha}^{n} b_{\alpha}^{0} \int_{0}^{t^{[0]}} u(s) d s+\left(\begin{array}{c}
n \\
1
\end{array}\right) a_{\alpha}^{n-1} b_{\alpha}^{1} \int_{0}^{t^{[1]}} u(s) d s \\
& +\cdots+\left(\begin{array}{c}
n \\
n-1
\end{array}\right) a_{\alpha}^{1} b_{\alpha}^{n-1} \int_{0}^{t^{[n-1]}} u(s) d s+\left(\begin{array}{c}
n \\
n
\end{array}\right) a_{\alpha}^{0} b_{\alpha}^{n} \int_{0}^{t^{[n]}} u(s) d s \\
= & \sum_{i=0}^{n}\left(\begin{array}{c}
n \\
i
\end{array}\right) a_{\alpha}^{n-i} b_{\alpha}^{i} \int_{0}^{t^{[i]}} u(s) d s \\
= & \sum_{i=0}^{n}\left(\begin{array}{c}
n \\
i
\end{array}\right) a_{\alpha}^{n-i} b_{\alpha}^{i} J^{i} u(t) .
\end{aligned}
$$

Below we present the main results of the manuscript.

Lemma 3.1 Let $u_{1}, v_{1} \in H^{1}(0,1)$ and $L$ a real number obeying $\left|u_{1}(s)-v_{1}(s)\right| \leq L$ for all $s \in[0,1]$. Thus, $\left.\right|^{C F} D^{\alpha^{[n]}} u_{1}(s)-{ }^{C F} D^{\alpha^{[n]}} v_{1}(s) \mid \leq \frac{(2-\alpha)^{n}}{(1-\alpha)^{2 n}} L$ for all $s \in[0,1]$. This result implies that $\left|{ }^{C F} D^{\alpha^{[n]}} u_{1}(s)\right| \leq \frac{(2-\alpha)^{n}}{(1-\alpha)^{2 n}} L$ for all $s \in[0,1]$ whenever $u_{1} \in H^{1}(0,1)$ with $\left|u_{1}(s)\right| \leq L$ for some $L \geq 0$ and all $s \in[0,1]$.

Lemma 3.2 Let $u_{1}, v_{1} \in H^{1}(0,1)$ with $u_{1}(0)=v_{1}(0)$ and La real number fulfilling $\mid u_{1}(s)$ $v_{1}(s) \mid \leq$ L for all $s \in[0,1]$. Thus, $\left|{ }^{C F} D^{\alpha^{[n]}} u_{1}(s)-{ }^{C F} D^{\alpha^{[n]}} v_{1}(s)\right| \leq \frac{1}{(1-\alpha)^{2 n}}$ L for all $s \in[0,1]$. This result implies that $\left|{ }^{C F} D^{\alpha^{[n]}} u_{1}(s)\right| \leq \frac{1}{(1-\alpha)^{2 n}} L$ for all $s \in[0,1]$ whenever $u_{1} \in H^{1}(0,1)$ with $u_{1}(0)=0$ and $\left|u_{1}(s)\right| \leq L$ for some $L \geq 0$ and all $s \in[0,1]$. 
Lemma 3.3 Let $u_{1}, v_{1} \in C_{\mathbb{R}}[0,1]$ and there is $L \geq 0$ satisfying $\left|u_{1}(s)-v_{1}(s)\right| \leq L$ for all $s \in$ $[0,1]$. Thus, $\left|{ }^{C F} I^{\alpha[n]} u_{1}(s)-{ }^{C F} I^{\alpha^{[n]}} v_{1}(s)\right| \leq L$ for all $s \in[0,1]$.

This result implies that $\left.\right|^{C F} I^{\alpha^{[n]}} u_{1}(s) \mid \leq L$ for all $s \in[0,1]$ whenever $u \in C_{\mathbb{R}}[0,1]$ with $\left|u_{1}(s)\right| \leq L$ for some $L \geq 0$ and all $s \in[0,1]$.

Lemma 3.4 Let $0<\alpha<1$ and $u, v \in H^{1}(0,1)$. The problem ${ }^{C F} D^{\alpha^{[n]}} u(t)=v(t), u(0)=0$, possesses the following unique solution: $u(t)=\left(a_{\alpha}+b_{\alpha} J v(t)\right)^{[n]}$, where ${ }^{C F} D^{\alpha^{[n]}}$ is defined by $(*)$.

Proof By using the Lemma 2.1 for ${ }^{C F} D^{\alpha} u(t)=v(t)$, we get $u(t)=a_{\alpha} v(t)+b_{\alpha} \int_{0}^{t} v(s) d s$. Also by using Lemma 2.1 for ${ }^{C F} D^{\alpha^{[2]}} u(t)=v(t)$, we obtain ${ }^{C F} D^{\alpha} u(t)=a_{\alpha} v(t)+b_{\alpha} \int_{0}^{t} v(s) d s$. Hence,

$$
\begin{aligned}
u(t) & =a_{\alpha}\left(a_{\alpha} v(t)+b_{\alpha} \int_{0}^{t} v(s) d s\right)+b_{\alpha} \int_{0}^{t}\left(a_{\alpha} v(s)+b_{\alpha} \int_{0}^{s} v(r) d r\right) d s \\
& =a_{\alpha}^{2} v(t)+2 a_{\alpha} b_{\alpha} \int_{0}^{t} v(s) d s+b_{\alpha}^{2} \int_{0}^{t} \int_{0}^{s} v(r) d r d s \\
& =\left(a_{\alpha}+b_{\alpha} \int_{0}^{t} v(s) d s\right)^{[2]} .
\end{aligned}
$$

Suppose that $u(t)=\left(a_{\alpha}+b_{\alpha} J v(t)\right)^{[n]}$ is the solution of the equation ${ }^{C F} D^{\alpha^{[n]}} u(t)=v(t)$. We show that $u(t)=\left(a_{\alpha}+b_{\alpha} J v(t)\right)^{[n+1]}$ is the solution of the equation ${ }^{C F} D^{\alpha{ }^{[n+1]}} u(t)=$ $v(t)$.

$$
\text { If } \begin{aligned}
&{ }^{C F} D^{\alpha[n]}\left({ }^{C F} D^{\alpha} u(t)\right)=v(t) \text {, then }{ }^{C F} D^{\alpha} u(t)=\left(a_{\alpha}+b_{\alpha} J v(t)\right)^{[n]} \text {. Thus, } \\
& u(t)= a_{\alpha}\left(a_{\alpha}+b_{\alpha} J v(t)\right)^{[n]}+b_{\alpha} \int_{0}^{t}\left(a_{\alpha}+b_{\alpha} J v(s)\right)^{[n]} d s \\
&= a_{\alpha}\left[\left(\begin{array}{l}
n \\
0
\end{array}\right) a_{\alpha}^{n} b_{\alpha}^{0} \int_{0}^{t^{[0]}} v(s) d s+\left(\begin{array}{l}
n \\
1
\end{array}\right) a_{\alpha}^{n-1} b_{\alpha}^{1} \int_{0}^{t^{[1]}} v(s) d s\right. \\
&\left.+\cdots+\left(\begin{array}{c}
n \\
n-1
\end{array}\right) a_{\alpha}^{1} b_{\alpha}^{n-1} \int_{0}^{t^{[n-1]}} v(s) d s+\left(\begin{array}{l}
n \\
n
\end{array}\right) a_{\alpha}^{0} b_{\alpha}^{n} \int_{0}^{t^{[n]}} v(s) d s\right] \\
&+b_{\alpha}\left[\left(\begin{array}{c}
n \\
0
\end{array}\right) a_{\alpha}^{n} b_{\alpha}^{0} \int_{0}^{t^{[1]}} v(s) d s+\left(\begin{array}{l}
n \\
1
\end{array}\right) a_{\alpha}^{n-1} b_{\alpha}^{1} \int_{0}^{t^{[2]}} v(s) d s\right. \\
&\left.+\cdots+\left(\begin{array}{c}
n \\
n-1
\end{array}\right) a_{\alpha}^{1} b_{\alpha}^{n-1} \int_{0}^{t^{[n]}} v(s) d s+\left(\begin{array}{l}
n \\
n
\end{array}\right) a_{\alpha}^{0} b_{\alpha}^{n} \int_{0}^{t^{[n+1]}} v(s) d s\right] \\
&=\left(\begin{array}{c}
n \\
0
\end{array}\right) a_{\alpha}^{n+1} b_{\alpha}^{0} \int_{0}^{t^{[0]}} v(s) d s+\left[\left(\begin{array}{c}
n \\
1
\end{array}\right)+\left(\begin{array}{l}
n \\
0
\end{array}\right)\right] a_{\alpha}^{n} b_{\alpha}^{1} \int_{0}^{t^{[1]}} v(s) d s \\
&+\cdots+\left[\left(\begin{array}{l}
n \\
n
\end{array}\right)+\left(\begin{array}{c}
n \\
n-1
\end{array}\right)\right] a_{\alpha}^{1} b_{\alpha}^{n} \int_{0}^{t^{[n]}} v(s) d s+\left(\begin{array}{l}
n \\
n
\end{array}\right) a_{\alpha}^{0} b_{\alpha}^{n+1} \int_{0}^{t^{[n+1]}} v(s) d s \\
&=\left(\begin{array}{c}
n+1 \\
0
\end{array}\right) a_{\alpha}^{n+1} b_{\alpha}^{0} \int_{0}^{t^{[0]}} v(s) d s+\left(\begin{array}{c}
n+1 \\
1
\end{array}\right) a_{\alpha}^{n} b_{\alpha}^{1} \int_{0}^{t^{[1]}} v(s) d s
\end{aligned}
$$




$$
\begin{aligned}
& +\cdots+\left(\begin{array}{c}
n+1 \\
n
\end{array}\right)+a_{\alpha}^{1} b_{\alpha}^{n} \int_{0}^{t^{[n]}} v(s) d s \\
& +\left(\begin{array}{c}
n+1 \\
n+1
\end{array}\right) a_{\alpha}^{0} b_{\alpha}^{n+1} \int_{0}^{t^{[n+1]}} v(s) d s \\
& =\left(a_{\alpha}+b_{\alpha} J v(t)\right)^{[n+1]}
\end{aligned}
$$

and so $u(t)=\left(a_{\alpha}+b_{\alpha} \int_{0}^{t} v(s) d s\right)^{[n]}=\left(a_{\alpha}+b_{\alpha} J v(t)\right)^{[n]}$ holds for all $n$.

In the last result, we used some notation such as $\int_{0}^{t^{[n]}} u(s) d s$, which was introduced by $(* *)$. We need the following result.

Lemma 3.5 ([17]) Suppose $t \in \mathbb{R}$, then $e^{t}=\sum_{i=0}^{\infty} \frac{t^{i}}{i !}$ for $0<|t|<\infty, t \Pi_{i=1}^{\infty}\left(1-\frac{t^{2}}{i^{2} \pi^{2}}\right)=\sin t$ and $\Pi_{i=1}^{\infty}\left(1-\frac{4 t^{2}}{(2 i-1)^{2} \pi^{2}}\right)=\cos t$.

Let $\gamma, \lambda:[0,1] \times[0,1] \rightarrow[0, \infty)$ denoting two continuous maps with $\sup _{r \in I}\left|\int_{0}^{t} \lambda(r, s) d s\right|<\infty$ and $\sup _{r \in I}\left|\int_{0}^{r} \gamma(r, s) d s\right|<\infty$, respectively.

Let $\phi$ and $\varphi$ be two maps defined as $(\phi u)(r)=\int_{0}^{r} \gamma(r, s) u(s) d s$ and $(\varphi u)(r)=$ $\int_{0}^{r} \lambda(r, s) u(s) d s$, respectively. Let $\eta \in L^{\infty}(I)$ with $\eta^{*}=\sup _{t \in I}|\eta(t)|$ and $k, h$ and $g$ be continuous on [0,1] with $M_{1}=\sup _{t \in I}|k(t)|, M_{2}=\sup _{t \in I}|h(t)|$ and $M_{3}=\sup _{t \in I}|g(t)|$. Put $\gamma_{0}=\sup \left|\int_{0}^{t} \gamma(t, s) d s\right|$ and $\lambda_{0}=\sup \left|\int_{0}^{t} \lambda(t, s) d s\right|$. Below we study the fractional-order integro-differential problem

$$
\begin{aligned}
{ }^{C F} D^{\alpha^{[n]}} z(s)= & \mu k(s){ }^{C F} D^{\beta^{[m]}}\left(z(s)+h(s){ }^{C F} D^{\gamma^{[p]}} z(s)\right) \\
& +f\left(s, z(s),(\phi z)(s),(\varphi z)(s),{ }^{C F} I^{\theta[q]} z(s), g(s){ }^{C F} D^{\delta^{[r]}} z(s)\right)
\end{aligned}
$$

with $z(0)=0$ under some conditions, where $\mu>1$ and $\alpha, \beta, \gamma, \theta, \delta \in(0,1)$ as well as $n, m, p, q, r \geq 1$. Since ${ }^{C F} D^{\alpha^{[n]}} u \in H^{1}$ for all $n$, the right hand is too.

Theorem 3.6 Let $f:[0,1] \times \mathbb{R}^{5} \rightarrow \mathbb{R}$ be a continuous function such that

$$
\begin{aligned}
& \left|f\left(t_{1}, x_{1}, y_{1}, w_{1}, u_{1}, u_{2}\right)-f\left(t_{1}, x_{1}^{\prime}, y_{1}^{\prime}, w_{1}^{\prime}, v_{1}, v_{2}\right)\right| \\
& \quad \leq \eta\left(t_{1}\right)\left(\left|x_{1}-x_{1}^{\prime}\right|+\left|y_{1}-y_{1}^{\prime}\right|+\left|w_{1}-w_{1}^{\prime}\right|+\left|u_{1}-v_{1}\right|+\left|u_{2}-v_{2}\right|\right)
\end{aligned}
$$

for all $t_{1} \in I$ and $x_{1}, y_{1}, w_{1}, x_{1}^{\prime}, y_{1}^{\prime}, w_{1}^{\prime}, u_{1}, u_{2}, v_{1}, v_{2} \in \mathbb{R}$. Then the stated problem (1) possesses an approximate solution when $\Delta=\eta^{*}\left(2+\gamma_{0}+\lambda_{0}+\frac{M_{3}}{(1-\delta)^{2 r}}\right)+\mu\left(\frac{M_{1} M_{2}}{(1-\gamma)^{2 p}(1-\beta)^{2 m}}+\frac{M_{1}}{(1-\beta)^{2 m}}\right)<1$.

Proof Let $H^{1}$ equipped with $d(z, v)=\|z-v\|$ on $X$, such that $\|z\|=\sup _{t \in I}|z(t)|$. Let $F$ : $H^{1} \rightarrow H^{1}$ be a map defined as follows:

$$
\begin{aligned}
(F z)(t)= & \left(a_{\alpha}+b_{\alpha} \int_{0}^{t}\left[\mu k(s)^{C F} D^{\beta^{[m]}}\left(z(s)+h(s)^{C F} D^{\gamma^{[p]}} z(s)\right)\right.\right. \\
& \left.\left.+f\left(s, z(s),(\phi z)(s),(\varphi z)(s),{ }^{C F} I^{\theta[q]} z(s), g(s)^{C F} D^{\delta^{[r]}} z(s)\right)\right] d s\right)^{[n]},
\end{aligned}
$$


where $a_{\alpha}$ and $b_{\alpha}$ are introduced in Lemma 2.1 and the notation ${ }^{C F} I^{\theta[q]} z(s)$ and ${ }^{C F} D^{\gamma[p]} z(s)$ is introduced by $(*)$ and $(* *)$. By using Lemmas 3.2 and 3.3 , we get

$$
\begin{aligned}
& \mid \mu k(s)^{C F} D^{\beta^{[m]}}\left(z(s)+h(s)^{C F} D^{\gamma^{[p]}} z(s)\right) \\
& +f\left(s, z(s),(\phi z)(s),(\varphi z)(s),{ }^{C F} I^{\theta[q]} z(s), g(s){ }^{C F} D^{\delta[r]} z(s)\right) \\
& -\mu k(s)^{C F} D^{\beta^{[m]}}\left(v(s)+h(s)^{C F} D^{\gamma^{[p]}} v(s)\right) \\
& -f\left(s, v(s),(\phi v)(s),(\varphi v)(s),{ }^{C F} I^{\theta^{[q]}} v(s), g(s)^{C F} D^{\delta^{[r]}} v(s)\right) \mid \\
& \leq \mu|| k(s)\left|{ }^{C F} D^{\beta^{[m]}}\left(z(s)+h(s)^{C F} D^{\gamma^{[p]}} z(s)\right)-{ }^{C F} D^{\beta^{[m]}}\left(v(s)+h(s)^{C F} D^{\gamma^{[p]}} v(s)\right)\right| \\
& +\mid f\left(s, z(s),(\phi z)(s),(\varphi z)(s),{ }^{C F} I^{\theta^{[q]}} z(s), g(s)^{C F} D^{\delta[r]} z(s)\right) \\
& -f\left(s, v(s),(\phi v)(s),(\varphi v)(s),{ }^{C F} I^{\theta^{[q]}} v(s), g(s)^{C F} D^{\delta[r]} v(s)\right) \mid \\
& \leq \mu\left[\left.|k(s)|\right|^{C F} D^{\beta^{[m]}}(z(s)-v(s)) \mid\right. \\
& \left.+|k(s)||h(s)|^{C F} D^{\beta^{[m]}}\left({ }^{C F} D^{\gamma^{[p]}}(z(s)-v(s))\right)\right] \\
& +|\eta(s)|[|z(s)-v(s)|+|(\phi z)(s)-(\phi v)(s)| \\
& \left.+|(\varphi z)(s)-(\varphi v)(s)|+\left|{ }^{C F} I^{\theta^{[q]}} z(s)-{ }^{C F} I^{\theta^{[q]}} v(s)\right|+|g(s)|\left|{ }^{C F} D^{\delta^{[r]}} z(s)-{ }^{C F} D^{\delta^{[r]}} v(s)\right|\right] \\
& \leq\left[\eta^{*}\left(2+\gamma_{0}+\lambda_{0}+\frac{M_{3}}{(1-\delta)^{2 r}}\right)+\mu\left(\frac{M_{1} M_{2}}{(1-\gamma)^{2 p}(1-\beta)^{2 m}}+\frac{M_{1}}{(1-\beta)^{2 m}}\right)\right]\|z-v\| .
\end{aligned}
$$

Since $z(0)=0$, we obtain

$$
\begin{aligned}
& |(F z)(t)-(F v)(t)| \\
& \leq\left(a_{\alpha}+b_{\alpha} \int_{0}^{t}\left[\eta^{*}\left(2+\gamma_{0}+\lambda_{0}+\frac{M_{3}}{(1-\delta)^{2 r}}\right)\right.\right. \\
& \left.\left.\quad+\mu\left(\frac{M_{1} M_{2}}{(1-\gamma)^{2 p}(1-\beta)^{2 m}}+\frac{M_{1}}{(1-\beta)^{2 m}}\right)\right]\|z-v\| d s\right)^{[n]}
\end{aligned}
$$

and so

$$
\begin{aligned}
& \|F z-F v\| \\
& \quad \leq\left(a_{\alpha}+b_{\alpha}\right)^{n}\left[\eta^{*}\left(2+\gamma_{0}+\lambda_{0}+\frac{M_{3}}{(1-\delta)^{2 r}}\right)\right. \\
& \left.\quad+\mu\left(\frac{M_{1} M_{2}}{(1-\gamma)^{2 p}(1-\beta)^{2 m}}+\frac{M_{1}}{(1-\beta)^{2 m}}\right)\right]\|z-v\|
\end{aligned}
$$

for all $t \in I$ and $z, v \in H^{1}$. Define the mappings $g:[0, \infty)^{5} \rightarrow[0, \infty)$ and $\alpha: H^{1} \times H^{1} \rightarrow$ $[0, \infty)$ by $g\left(x_{1}, x_{2}, x_{3}, x_{4}, x_{5}\right)=\frac{\Delta}{9}\left(3 x_{1}+2 x_{2}+4 x_{3}\right)$ and $\alpha(x, y)=1$ for all $x, y \in H^{1}$ and $x_{1}, \ldots, x_{5} \in[0, \infty)$. One can easily check that $g \in \mathcal{R}$. In addition we conclude that $F$ denotes a generalized $\alpha$-contractive map. From Theorem 2.2, we conclude that $F$ possesses an approximate fixed point which is an approximate solution of (1).

Suppose that functions $k, s, h, g$ and $q$ are bounded on [0,1] with $M_{1}=\sup _{t \in I}|k(t)|<$ $\infty, M_{2}=\sup _{t \in I}|s(t)|<\infty, M_{3}=\sup _{t \in I}|h(t)|<\infty, M_{4}=\sup _{t \in I}|g(t)|<\infty$ and $M_{5}=$ 
$\sup _{t \in I}|q(t)|<\infty$. Now we discus the following problem:

$$
\begin{aligned}
{ }^{C F} D^{\alpha^{[n]}} u(r)= & \lambda k(r){ }^{C F} D^{\beta^{[m]}} u(r)+\mu s(r)^{C F} I^{\rho^{[p]}} u(r) \\
& +\sum_{i=0}^{\infty} \frac{{ }^{C F} D^{\theta^{[i]}} f_{1}\left(r, u(r),(\phi u)(r), h(r)^{C F} I^{[q]} u(r), g(r){ }^{C F} D^{\delta^{[r]}} u(r)\right)}{i !} \\
& +\int_{0}^{r} f_{2}\left(s, u(s),(\varphi u)(s), q(s) \sum_{i=0}^{\infty} \frac{C F D^{\gamma^{[i]}} u(s)}{d^{i}}\right) d s
\end{aligned}
$$

with $u(0)=0$ under some conditions, where $\lambda, \mu \geq 0, \alpha, \beta, \rho, \theta, \nu, \delta \in(0,1),\left|\frac{1}{d(1-\gamma)^{2}}\right|<1$ and $n, m, p, q, r, k \geq 1$. The functions $k, s, h, g$ and $q$ maybe are not continuous, but the right hand of (2) should be a member of $H^{1}$ because ${ }^{C F} D^{\alpha^{[n]}} u \in H^{1}$.

Theorem 3.7 Suppose that $f_{1}:[0,1] \times \mathbb{R}^{4} \rightarrow \mathbb{R}$ and $f_{2}:[0,1] \times \mathbb{R}^{3} \rightarrow \mathbb{R}$ are integrable functions such that

$$
\begin{aligned}
& \left|f_{1}\left(t_{1}, x_{1}, y_{1}, w_{1}, v_{1}\right)-f_{1}\left(t, x_{1}^{\prime}, y_{1}^{\prime}, w_{1}^{\prime}, v_{1}^{\prime}\right)\right| \\
& \quad \leq \xi_{1}\left|x_{1}-x_{1}^{\prime}\right|+\xi_{2}\left|y_{1}-y_{1}^{\prime}\right|+\xi_{3}\left|w_{1}-w_{1}^{\prime}\right|+\xi_{4}\left|v_{1}-v_{1}^{\prime}\right|, \\
& \left|f_{2}\left(t_{1}, x_{1}, y_{1}, w_{1}\right)-f_{2}\left(t_{1}, x_{1}^{\prime}, y_{1}^{\prime}, w_{1}^{\prime}\right)\right| \\
& \quad \leq \xi_{1}^{\prime}\left|x_{1}-x_{1}^{\prime}\right|+\xi_{2}^{\prime}\left|y_{1}-y_{1}^{\prime}\right|+\xi_{3}^{\prime}\left|w_{1}-w_{1}^{\prime}\right|
\end{aligned}
$$

for some nonnegative real numbers $\lambda, \mu, \xi_{1}, \xi_{2}, \xi_{3}, \xi_{4}, \xi_{1}^{\prime}, \xi_{2}^{\prime}, \xi_{3}^{\prime}$ and all $x_{1}, y_{1}, w_{1}, v_{1}, x_{1}^{\prime}, y_{1}^{\prime}, w_{1}^{\prime}$, $v_{1}^{\prime} \in \mathbb{R}$ and $t \in$ I. If $\Delta=\left[\lambda \frac{M_{1}}{(1-\beta)^{2 m}}+\mu M_{2}+e^{\frac{1}{(1-\theta)^{2}}}\left(\xi_{1}+\xi_{2} \gamma_{0}+\xi_{3} M_{3}+\xi_{4} \frac{M_{4}}{(1-\delta)^{2 r}}\right)+\xi_{1}^{\prime}+\xi_{2}^{\prime} \lambda_{0}+\right.$ $\left.\frac{\xi_{3}^{\prime} d(1-\gamma)^{2} M_{5}}{d(1-\gamma)^{2}-1}\right]<1$, then the stated problem (2) possesses an approximate solution.

Proof Let $H^{1}$ equipped with $d(z, v)=\|z-v\|$, such that $\|z\|=\sup _{t \in I}|z(t)|$. Define the map $F: H^{1} \rightarrow H^{1}$ by

$$
\begin{aligned}
(F z)(t)= & \left(a_{\alpha}+b_{\alpha} \int_{0}^{t}\left[\lambda k(s)^{C F} D^{\beta^{[m]}} z(s)+\mu s(s)^{C F} I^{\rho^{[p]}} z(s)\right.\right. \\
& +\sum_{i=0}^{\infty} \frac{{ }^{C F} D^{\theta^{[i]}} f_{1}\left(s, z(s),(\phi z)(s), h(s)^{C F} I^{\nu^{[q]}} z(s), g(s)^{C F} D^{\delta[r]} z(s)\right)}{i !} \\
& \left.\left.+\int_{0}^{s} f_{2}(r, z(r),(\varphi z)(r), q(r)) \sum_{i=0}^{\infty} \frac{{ }^{C F} D^{\gamma^{[i]}} z(r)}{d^{i}} d r\right] d s\right)^{[n]} .
\end{aligned}
$$

By using Lemmas 3.2, 3.3 and 3.5, we get

$$
\begin{aligned}
& \|\left[\lambda k(s)^{C F} D^{\beta^{[m]} z(s)+\mu s(s)^{C F} I^{\rho[p]} z(s)}\right. \\
& \quad+\sum_{i=0}^{\infty} \frac{{ }^{C F} D^{\theta^{[i]}} f_{1}\left(s, z(s),(\phi z)(s), h(s)^{C F} I^{[[q]} z(s), g(s)^{C F} D^{\delta[r]} z(s)\right)}{i !} \\
& \quad+\int_{0}^{s} f_{2}\left(r, z(r),(\varphi z)(r), q(r) \sum_{i=0}^{\infty} \frac{{ }^{C F} D^{\gamma^{[i]}} z(r)}{2^{i}} d r\right]
\end{aligned}
$$




$$
\begin{aligned}
& -\left[\lambda k(s)^{C F} D^{\beta^{[m]}} v(s)+\mu s(s)^{C F} I^{\rho^{[p]}} v(s)\right. \\
& +\sum_{i=0}^{\infty} \frac{{ }^{C F} D^{\theta^{[i]}} f_{1}\left(s, v(s),(\phi v)(s), h(s)^{C F} I^{\nu^{[q]}} v(s), g(s)^{C F} D^{\delta^{[r]}} v(s)\right)}{i !} \\
& +\int_{0}^{r} f_{2}\left(r, v(r),(\varphi v)(r), q(r) \sum_{i=0}^{\infty} \frac{{ }^{C F} D^{\gamma^{[i]}} v(r)}{d^{i}} d r\right] \mid \\
& \leq\left.\lambda|k(s)|\right|^{C F} D^{\beta^{[m]}}(z(s)-v(s))|+\mu| s(s)||^{C F} I^{\rho^{[p]}}(z(s)-v(s)) \mid \\
& +\mid \sum_{i=0}^{\infty} \frac{1}{i !(1-\theta)^{2 i}}\left[f_{1}\left(s, z(s),(\phi z)(s), h(s)^{C F} I^{v^{[q]}} z(s), g(s)^{C F} D^{\delta^{[r]}} z(s)\right)\right. \\
& \left.-f_{1}\left(s, v(s),(\phi v)(s), h(s)^{C F} I^{v[q]} v(s), g(s)^{C F} D^{\delta^{[r]}} v(s)\right)\right] \\
& +\int_{0}^{s} \mid f_{2}\left(r, z(r),(\varphi z)(r), q(r) \sum_{i=0}^{\infty} \frac{{ }^{C F} D^{\gamma^{[i]}} z(r)}{d^{i}}\right. \\
& -f_{2}\left(r, v(r),(\varphi v)(r), q(r) \sum_{i=0}^{\infty} \frac{{ }^{C F} D^{\gamma^{[i]}} v(r)}{d^{i}} \mid d r\right. \\
& \leq \lambda \frac{M_{1}}{(1-\beta)^{2 m}}\|z-v\|+\mu M_{2}\|z-v\| \\
& +\sum_{i=0}^{\infty} \frac{1}{i !(1-\theta)^{2 i}}\left(\xi_{1}\|z-v\|+\xi_{2} \gamma_{0}\|z-v\|+\xi_{3} M_{3}\|z-v\|+\xi_{4} \frac{M_{4}}{(1-\delta)^{2 r}}\|z-v\|\right) \\
& +\xi_{1}^{\prime}\|z-v\|+\xi_{2}^{\prime} \lambda_{0}\|z-v\|+\sum_{i=0}^{\infty} \frac{\xi_{3}^{\prime} M_{5}}{d^{i}(1-\gamma)^{2 i}}\|z-v\| \\
& =\left[\lambda \frac{M_{1}}{(1-\beta)^{2 m}}+\mu M_{2}+e^{\frac{1}{(1-\theta)^{2}}}\left(\xi_{1}+\xi_{2} \gamma_{0}+\xi_{3} M_{3}+\xi_{4} \frac{M_{4}}{(1-\delta)^{2 r}}\right)\right. \\
& \left.+\xi_{1}^{\prime}+\xi_{2}^{\prime} \lambda_{0}+\frac{\xi_{3}^{\prime} d(1-\gamma)^{2} M_{5}}{d(1-\gamma)^{2}-1}\right]\|z-v\| \\
& =\Delta\|z-v\| \text {. }
\end{aligned}
$$

Since $z(0)=v(0),|(F z)(t)-(F v)(t)| \leq\left(a_{\alpha}+b_{\alpha} \int_{0}^{t} \Delta\|z-v\| d s\right)^{[n]}$. Hence, $\|F z-F v\| \leq \Delta\|z-v\|$ for all $t \in I$ and $z, v \in H^{1}$. Define the mappings $g:[0, \infty)^{5} \rightarrow[0, \infty)$ and $\alpha: H^{1} \times H^{1} \rightarrow$ $[0, \infty)$ by $g\left(x_{1}, x_{2}, x_{3}, x_{4}, x_{5}\right)=\frac{\Delta}{9}\left(3 x_{1}+2 x_{2}+4 x_{3}\right)$ and $\alpha(x, y)=1$ for all $z, v \in H^{1}$ and $x_{1}, \ldots, x_{5} \in[0, \infty)$. One can easily check that $g \in \mathcal{R}$. By simple calculations we prove that $F$ represents a generalized $\alpha$-contractive map. Besides, from Theorem 2.2, we conclude that $F$ possesses an approximate fixed point which represents an approximate solution for (2).

Below we show two illustrative examples.

Example 3.1 Let the maps $\eta_{1} \in L^{\infty}([0,1])$ and $\gamma_{1}, \lambda_{1}:[0,1] \times[0,1] \rightarrow[0, \infty)$ be $\eta_{1}(t)=$ $\frac{e^{-(\pi t+16)}}{2}, \gamma_{1}(t, s)=e^{t-s}$ and $\lambda_{1}(t, s)=e^{\ln (|2 t-s|+1)}$. Then $\eta^{*}=\frac{1}{2 e^{16}}, \gamma_{0} \leq e$ and $\lambda_{0} \leq e^{\ln (3)}$. Now, put $\mu=\frac{1}{e^{16}}, \alpha=\frac{1}{3}, \beta=\frac{1}{4}, \gamma=\frac{3}{5}, \theta=\frac{6}{7}, \delta=\frac{1}{2}, n=61, m=3, p=2, q=57$ and $r=$ 
73. Let $k_{1}(t)=\sin (t), h_{1}(t)=\frac{t-2}{2 t+1}$ and $g_{1}(t)=\frac{1}{2^{141}} \tan ^{-1}(t)$ be two functions. Then $M_{1}=$ $\sup _{t \in I}\left|k_{1}(t)\right|=1, M_{2}=\sup _{t \in I}\left|h_{1}(t)\right|=2$ and $M_{3}=\sup _{t \in I}\left|g_{1}(t)\right|=\frac{\pi}{2^{142}}$. Let us discus

$$
\begin{aligned}
{ }^{C F} D^{\frac{1}{3}}{ }^{[6]]} u(t)= & \frac{1}{e^{16}} \sin t^{C F} D^{\frac{1}{4}}{ }^{[3]}\left(u(t)+\frac{t-2}{2 t+1}{ }^{C F} D^{\frac{3}{5}}{ }^{[2]} u(t)\right) \\
& +\frac{e^{-(\pi t+16)}}{2}\left[2 t+\frac{1}{8} u(t)+\frac{5}{11} \int_{0}^{t} e^{t-s} u(s) d s+\int_{0}^{t} e^{\ln (|2 t-s|+1)} u(s) d s\right. \\
& \left.\left.+\frac{1}{e^{6}}{ }^{C F} I^{\frac{6}{7}}{ }^{[57]} u(t)+\frac{1}{2^{141}} \tan ^{-1}(t)^{C F} D^{\frac{1}{2}^{[73]}} u(t)\right)\right]
\end{aligned}
$$

with $u(0)=0$. Let $f\left(t_{1}, x_{1}, y_{1}, w_{1}, u_{1}, u_{2}\right)=\frac{e^{-\left(\pi t_{1}+6\right)}}{2}\left(2 t+\frac{1}{8} x_{1}+\frac{5}{11} y_{1}+w_{1}+e^{-6} u_{1}+u_{2}\right)$. In our case $\Delta=\left[\eta^{*}\left(2+\gamma_{0}+\lambda_{0}+\frac{M_{3}}{(1-\delta)^{2 r}}\right)+\mu\left(\frac{M_{1} M_{2}}{(1-\gamma)^{2 p}(1-\beta)^{2 m}}+\frac{M_{1}}{(1-\beta)^{2 m}}\right)\right]<0.0374<1$. Now by using Theorem 3.6, (3) has an approximate solution.

Example 3.2 Let $\gamma_{1}, \lambda_{1}:[0,1] \times[0,1] \rightarrow[0, \infty)$ be $\gamma_{1}(t, s)=\frac{t-s}{1+2 t}$ and $\lambda_{1}(t, s)=\sin (t-$ $s) e^{\ln (|2 t-s|+1)}$, respectively. Then $\gamma_{0} \leq 1$ and $\lambda_{0} \leq e^{\ln (3)}$. Put $\lambda=\frac{2}{2,037}, \mu=\frac{2}{421}, \alpha=\frac{1}{4}, \beta=\frac{1}{2}$, $\rho=\frac{1}{2}, \theta=\frac{1}{2}, v=\frac{1}{4}, \delta=\frac{1}{4}, \gamma=\frac{1}{2}, n=7, m=3, p=2, q=3, r=3, d=12, \xi_{1}=\frac{3}{6,041}$, $\xi_{2}=\frac{1}{5,920}, \xi_{3}=\frac{1}{803}, \xi_{4}=\frac{1}{e^{18}}, \xi_{1}^{\prime}=\frac{2}{10^{6}}, \xi_{2}^{\prime}=\frac{e}{\pi^{11}}$ and $\xi_{3}^{\prime}=\frac{1}{600}$. Now, consider the functions $k(t)=\ln (2+t), s(t)=1, h(t)=1, g(t)=e^{\sin \pi t}, q(t)=\frac{1}{432}$ when $x \in \mathbb{Q} \cap[0,1]$ and $q(t)=0$ when $x \in \mathbb{Q}^{c} \cap[0,1]$. Then we have $M_{1}=\sup _{t \in I}|k(t)|=\ln 3, M_{2}=\sup _{t \in I}|s(t)|=1$, $M_{3}=\sup _{t \in I}|h(t)|=1, M_{4}=\sup _{t \in I}|g(t)|=e$ and $M_{5}=\sup _{t \in I}|q(t)|=\frac{1}{432}$. Now, consider the integro-differential problem

$$
\begin{aligned}
& { }^{C F} D^{1^{[}}{ }^{[7]} z(t)=\frac{2}{2,037} \ln (2+t)^{C F} D^{\frac{1}{2}^{[3]}} z(t)+\frac{2}{421}{ }^{C F} I^{\frac{1}{2}^{[2]}} z(t) \\
& +\sum_{i=0}^{\infty} \frac{1}{i !} C F D^{\frac{1}{2}[i]}\left(\frac{2}{91} t+\frac{3}{6,041} z(t)+\frac{1}{5,920} \int_{0}^{t} \frac{t-s}{1+2 t} d s\right. \\
& \left.+\frac{1}{803}{ }^{C F} I^{I^{\left[\frac{[3]}{4}\right.}} z(t)+\frac{1}{e^{18}} e^{\sin \pi t}(t)^{C F} D^{1^{[3]}} z(t)\right) \\
& +\int_{0}^{t}\left[s+\frac{2 z(s)}{10^{6}}+\frac{e}{\pi^{11}} \int_{0}^{s} \sin (t-s) e^{\ln (|2 s-r|+1)} z(r) d r\right. \\
& \left.+\frac{1}{600} q(s) \sum_{i=0}^{\infty} \frac{{ }^{C F} D^{1^{\left.\frac{1}{2}\right]}} z(s)}{12^{i}}\right] d s
\end{aligned}
$$

with $z(0)=0$. Let $f_{1}\left(t_{1}, x_{1}, y_{1}, w_{1}, v_{1}\right)=\frac{2}{91} t_{1}+\frac{3}{6,041} x_{1}+\frac{1}{5,920} y_{1}+\frac{1}{803} w_{1}+\frac{1}{e^{18}} v_{1}$ and $f_{2}\left(t_{1}, x_{1}, y_{1}\right.$, $\left.w_{1}\right)=t_{1}+\frac{2}{10^{6}} x_{1}+\frac{e}{\pi^{11}} y_{1}+\frac{1}{600} w_{1} 1$. In addition,

$$
\begin{aligned}
\Delta= & {\left[\lambda \frac{M_{1}}{(1-\beta)^{2 m}}+\mu M_{2}+e^{\frac{1}{(1-\theta)^{2}}}\left(\xi_{1}+\xi_{2} \gamma_{0}+\xi_{3} M_{3}+\xi_{4} \frac{M_{4}}{(1-\delta)^{2 r}}\right)\right.} \\
& \left.+\xi_{1}^{\prime}+\xi_{2}^{\prime} \lambda_{0}+\frac{\xi_{3}^{\prime} d(1-\gamma)^{2} M_{5}}{d(1-\gamma)^{2}-1}\right] \\
< & 0.179<1 .
\end{aligned}
$$

Now by using Theorem 3.7, the problem (4) possesses an approximate solution. 


\title{
4 Conclusions
}

The higher-order FDE play an important role in modelling the dynamics of complex systems. This direction is an important topic in modelling the dissipative phenomena especially by fractional derivatives as Riemann-Liouville and Caputo. However, the CF derivative is equipped with a non-singular kernel, therefore it was found attractive and very suitable for several types of models possessing a memory effect. Thus, finding suitable numerical techniques and their approximate solutions for some complicated models containing a CF higher-order derivative are subjects of current interest. Along this line of thought in this manuscript we show the existence of approximate solutions analytically for two higher-order Caputo-Fabrizio FDE. We check our results by providing two examples. We conclude this manuscript by saying that, utilizing the numerical methods, one can obtain approximations of the unknown exact solution.

\author{
Acknowledgements \\ Research of the third and fourth authors was supported by Azarbaijan Shahid Madani University. The authors express \\ their gratitude to the referees for their helpful suggestions.

\section{Competing interests} \\ The authors declare that they have no competing interests. \\ Authors' contributions \\ All authors read and approved the final manuscript.

\section{Author details} \\ 'Department of Mathematics, Isik University, Istanbul, Turkey. ${ }^{2}$ Department of Mathematics, Cankaya University, \\ Ogretmenler Cad. 14, Balgat, Ankara, 06530, Turkey. ${ }^{3}$ Institute of Space Sciences, Magurele, Bucharest, Romania. \\ ${ }^{4}$ Department of Mathematics, Azarbaijan Shahid Madani University, Tabriz, Iran.
}

\section{Publisher's Note}

Springer Nature remains neutral with regard to jurisdictional claims in published maps and institutional affiliations.

Received: 20 March 2017 Accepted: 29 June 2017 Published online: 03 August 2017

\section{References}

1. Alsaedi, A, Baleanu, D, Etemad, S, Rezapour, S: On coupled systems of time-fractional differential problems by using a new fractional derivative. J. Funct. Spaces 2016, Article ID 4626940 (2016)

2. Area, I, Losada, J, Nieto, JJ: A note on the fractional logistic equation. Physica A 444, 182-187 (2016)

3. Atangana, A: On the new fractional derivative and application to nonlinear Fisher's reaction-diffusion equation. Appl. Math. Comput. 273(6), 948-956 (2016)

4. Atangana, A, Alkahtani, BT: Analysis of the Keller-Segel model with a fractional derivative without singular kernel. Entropy 17(6), 4439-4453 (2015)

5. Baleanu, D, Hedayati, V, Rezapour, S, Al Qurashi, MM: On two fractional differential inclusions. SpringerPlus 5(1), 882 (2016)

6. Baleanu, D, Mousalou, A, Rezapour, S: A new method for investigating some fractional integro-differential equations involving the Caputo-Fabrizio derivative. Adv. Differ. Equ. 2017, 51 (2017)

7. De La Sen, M, Hedayati, V, Gholizade Atani, Y, Rezapour, S: The existence and numerical solution for a k-dimensional system of multi-term fractional integro-differential equations. Nonlinear Anal., Model. Control 22(2), 188-209 (2017)

8. Caputo, M, Fabrizzio, M: A new definition of fractional derivative without singular kernel. Prog. Fract. Differ. Appl. 1(2), 73-85 (2015)

9. Gómez-Aguilar, JF, Yépez-Martínez, H, Calderón-Ramón, C, Cruz-Orduña, I, Escobar-Jiménez, RF, Olivares-Peregrino, $\mathrm{VH}$ : Modeling of a mass-spring-damper system by fractional derivatives with and without a singular kernel. Entropy 17(9), 6289-6303 (2015)

10. Goufo, EF, Doungmo, P, Morgan, K, Mwambakana, JN: Duplication in a model of rock fracture with fractional derivative without singular kernel. Open Math. 13, 839-846 (2015)

11. Losada, J, Nieto, JJ: Properties of a new fractional derivative without singular kernel. Prog. Fract. Differ. Appl. 1(2), 87-92 (2015)

12. Miandaragh, MA, Postolache, M, Rezapour, S: Some approximate fixed point results for generalized $\alpha$-contractive mappings. U.P.B. Sci. Bull., Ser. A 75(2), 3-10 (2013)

13. Rezapour, S, Shabibi, M: A singular fractional differential equation with Riemann-Liouville integral boundary condition. J. Adv. Math. Stud. 8(1), 80-88 (2015)

14. Shabibi, M, Rezapour, S, Vaezpour, SM: A singular fractional integro-differential equation. U.P.B. Sci. Bull., Ser. A 79(1), 109-118 (2017) 
15. Shabibi, M, Postolache, M, Rezapour, S, Vaezpour, SM: Investigation of a multi-singular pointwise defined fractional integro-differential equation. J. Math. Anal. 7(5), 61-77 (2016)

16. Shabibi, M, Postolache, M, Rezapour, S: Positive solutions for a singular sum fractional differential system. Int. J. Anal. Appl. 13(1), 108-118 (2017)

17. Tspkin, AG, Tsypkin, GG: Mathematical Formulas. Mir, Moscow (1985)

Submit your manuscript to a SpringerOpen ${ }^{\circ}$ journal and benefit from:

- Convenient online submission

- Rigorous peer review

- Open access: articles freely available online

- High visibility within the field

- Retaining the copyright to your article

Submit your next manuscript at $\gg$ springeropen.com 